Research Paper

\title{
AMPK activation-dependent autophagy compromises oleanolic acid-induced cytotoxicity in human bladder cancer cells
}

\author{
Yarong Song ${ }^{1, *}$, Peng Zhang ${ }^{1, *}$, Yadong Sun ${ }^{1}$, Xuechao Li ${ }^{1}$, Lifeng Chen ${ }^{1}$, Yajun \\ $\mathrm{Xiao}^{1}$ and Yifei Xing ${ }^{1}$ \\ ${ }^{1}$ Department of Urology, Union Hospital, Tongji Medical College, Huazhong University of Science and Technology, Wuhan \\ 430022, China \\ "These authors contributed equally to this work
}

Correspondence to: Yifei Xing, email: yifei_xing@163.com

Keywords: bladder cancer, oleanolic acid, autophagy, AMPK, cytotoxicity

Received: October 22, $2016 \quad$ Accepted: June 16, $2017 \quad$ Published: July 04, 2017

Copyright: Song et al. This is an open-access article distributed under the terms of the Creative Commons Attribution License 3.0 (CC BY 3.0), which permits unrestricted use, distribution, and reproduction in any medium, provided the original author and source are credited.

\section{ABSTRACT}

Autophagy is an evolutionarily conserved catabolic process in eukaryotic cells, which allows cells to overcome a wide array of of stresses and has recently been shown to result in drug resistance. This study examined the effect of autophagy on oleanolic acid (OA)-induced cytotoxicity against bladder cancer cells. Our study demonstrated that $O A$ inhibited cell viability, proliferation, and induced apoptosis in bladder cancer lines T24 and EJ. Furthermore, OA induced autophagy in both cell lines by activating AMP-activated protein kinase (AMPK), inhibiting mechanistic target of rapamycin ( $m$ TOR) and promoting unc-51 like autophagy activating kinase 1 (ULK1). Moreover, inhibiting autophagy by siRNA to autophagy related 7 (ATG7) or with autophagy inhibitor bafilomycin A1 and 3-methyladenine (3-MA) or AMPK inhibitor dorsomorphin (compound C) promoted OA-induced deaths of bladder cancer cells. In contrast, either autophagy activator rapamycin or AMPK activator acadesine (AICAR) compromised OA-induced anti-cancer effect. Our findings suggested that OA induced protective autophagy through AMPK-mTOR-ULK1 signaling pathway in bladder cancer cells and $O A$ in combination with autophagy inhibitor might be a novel alternative for the treatment of bladder cancer.

\section{INTRODUCTION}

Bladder cancer represents one of the most common malignancies worldwide, with approximately 58,950 new cases and 11,820 deaths in the United State in 2015 [1]. Once bladder cancer becomes a muscle-invasive disease (MIBC), the risks for recurrence, progression and mortality will increase dramatically. For localized MIBC, radical cystectomy is routinely performed but the postoperative 5-year survival rate is only about $50 \%$ [2], and new and more effective therapeutic strategies are urgently needed.

Oleanolic acid (OA; 3 -hydroxyolean-12-en-28oic acid), a pentacyclic triterpene compound, has been isolated from more than 1620 plant species and has also been biosynthesized from lupane [3]. OA possesses a wide range of pharmacological properties, such as hepato- protective, anti-diabetic, anti-viral and anti-cancer effects [4], and it has been shown to be effective for the treatment of a variety of human cancers, such as breast cancer [5], gallbladder cancer [6], hepatocellular carcinoma, among others [7]. Our previous study demonstrated that OA effectively inhibited cell viability and proliferation and promoted cell apoptosis and cell cycle arrest at G0/G1 phase via PI3K/AKT pathway in human prostate cancer cells [8]. While an inhibitory effect on the proliferation of T24 cells was suggested [9], the exact underlying mechanism of OA working on bladder cancer has not been fully understood. Several studies have demonstrated that OA induced autophagy and promoted death pathway in cancer cells [10-13], but it is still unclear if autophagy is involved in OA-induced anti-cancer effect.

Autophagy is a highly conserved self-degrading process extensively found in eukaryotic cells and it 
begins from the formation of double-membrane vacuoles which sequester aggregate-prone proteins, organelles, or portions of the cytoplasm and infectious agents, and ends when they are delivered to the lysosome [14]. As a context-dependent mechanism, autophagy can not only suppress tumor growth but also promote the survival of tumor cells after pharmacotherapy and other intracellular or extracellular stresses [15]. On the one hand, autophagy plays an important role in maintaining cellular homeostasis, and its abnormality may disrupt cellular integrity and promote carcinogenesis. Autophagy was first found to be involved in tumor suppression since autophagic gene BECN1 bore relationship to several human cancers $[16,17]$. On the other hand, suppression of autophagy led to oxidative stress, a well-known cause of cancer initiation and progression [18, 19]. Due to increasing biosynthetic and metabolic demands imposed by deregulated proliferation, cancer cells are more dependent on autophagy $[15,20]$. Cancer cells exposed to cytotoxic agents may initiate protective autophagy by activating AMPK, GSK3 $\beta$, ERK1/2 and eEF2K pathways, and autophagy inhibition may enhance the cytotoxicity of anti-cancer agents [21].

In the present study, we investigated if OA induces cytotoxicity or autophagy in bladder cancer cells, and if autophagy, in turn, works on cytotoxicity. Our results revealed that OA induced both cytotoxicity and autophagy in T24 and EJ cells. Moreover, our study also showed that OA promoted autophagy by triggering phosphorylation of AMPK $\alpha$ Thr172, thereby inhibiting mTOR and activating ULK1. Furthermore, we found that inhibition of autophagy enhanced OA-induced cytotoxicity, and on the contrary, induction of autophagy abolished such effects. To sum up, our data suggested that autophagy exerts a cytoprotective effect on bladder cancer cells by compromising OAmediated anti-cancer effect. These findings indicated that $\mathrm{OA}$ in combination with autophagy inhibitors might be a more effective therapeutic alternative for bladder cancer.

\section{RESULTS}

\section{OA inhibited viability and proliferation of human bladder cancer cells}

To determine whether OA inhibits viability and proliferation of human bladder cancer cells, T24 and EJ cells were treated with OA for $24 \mathrm{~h}$. The CCK- 8 assay showed that OA treatment decreased cell viability in both cells in a dose-dependent manner (Figure 1A). On the basis of this finding, we chose 7.5 and $15 \mu \mathrm{M}$ as the appropriate concentration for both cell lines in subsequent experiments. Colony formation assay was applied to evaluate the ability of T24 and EJ cells to form colonies at various different concentrations of OA (Figure 1B) and the results indicated that $\mathrm{OA}$ suppressed colony formation dose-dependently in both cell lines (Figure 1C). These findings suggested that OA could inhibit the viability and proliferation of human bladder cancer cells.

\section{OA induced apoptosis in human bladder cancer cells}

In order to know whether OA promotes apoptosis in human bladder cancer cells, T24 and EJ cells were incubated with or without 7.5 and $15 \mu \mathrm{M}$ of OA. The cells were then stained with Annexin V/FITC and PI, and then flow cytometrically examined. As shown in Figure 2A and $2 \mathrm{~B}$, in both cell lines, $\mathrm{OA}$ treatment increased the number of Annexin V-positive cells in a does-dependent manner. To further understand the mechanisms of OA inducing apoptosis, the expression of some apoptosisassociated proteins were detected, and result revealed that OA activated pro-apoptotic Bax and cleaved caspase-3, while reduced expression of anti-apoptotic Bcl-2 (Figure 2C). These results suggested that OA-induced apoptosis in human bladder cancer cells by controlling of apoptosis regulators.

\section{OA boosted autophagy in human bladder cancer cells}

Since autophagy is an evolutionarily conserved process in response to a variety of stresses including anti-cancer stimuli and plays either a cytopathic or cytoprotective role [22], we investigated whether OA induces autophagy in human bladder cancer cells. First, we transfected cells with adenovirus carrying LC3B (mCherry-GFP-LC3B) for $24 \mathrm{~h}$ and then treated the cells with or without OA for another $12 \mathrm{~h}$. Under normal growth conditions, both green (GFP) and red (mCherry) fluorescence diffusely distribute predominantly in the nucleus and cytoplasm. When cells were exposed to various autophagic stimuli, Staining of LC3B with these two fluorescent proteins showed that autophagosome membranes contained LC3B puncta (yellow fluorescentce). Autophagolysosomes displayed only red fluorescent signal (mCherry), which is relatively stable, while GFP fluorescence quenched under the acidic conditions of the lysosome lumen. After treatment with $7.5 \mu \mathrm{M}$ of $\mathrm{OA}$, T24 and EJ cells were observed under a fluorescence microscope and images were processed with cellSens Entry. Figure $3 \mathrm{~A}$ and $3 \mathrm{~B}$ shows that, after OA treatment, the number of yellow puncta (both red- and green-stained, $\mathrm{R}+\mathrm{G}^{+}$) and red puncta (red- but not green-stained, $\mathrm{R}+\mathrm{G}^{-}$) increased remarkably as compared with the negative control groups. Moreover, we also examined LC3B-II, the lipidized form of LC3B modified from LC3B-I by Western blotting and results showed that LC3B-II level increased gradually in both T24 and EJ cells in a dose-dependent fashion after OA treatment (Figure 3C). These results were further verified by transmission electron microscopy (TEM), which revealed that there existed electron lucent 
vacuoles in bladder cancer cells following $7.5 \mu \mathrm{M}$ OA treatments. Finally, autophagosomes were distinctly different from empty vacuoles in that, under higher magnifications, autophagosomes had a double-membrane containing cellular materials and highly electrondense lysosomal structures (Figure 3D). These findings collectively proved that OA could induce autophagy in human bladder cancer cells.

\section{OA activated AMPK-mTOR-ULK1 axis in human bladder cancer cells}

AMPK is a cellular energy sensor that can be triggered by nutrient starvation or other naturally occurring compounds, thus promoting autophagy [23-25]. To further determine the molecular mechanisms of OA inducing autophagy, the levels of AMPK $\alpha$ and P-AMPK $\alpha$
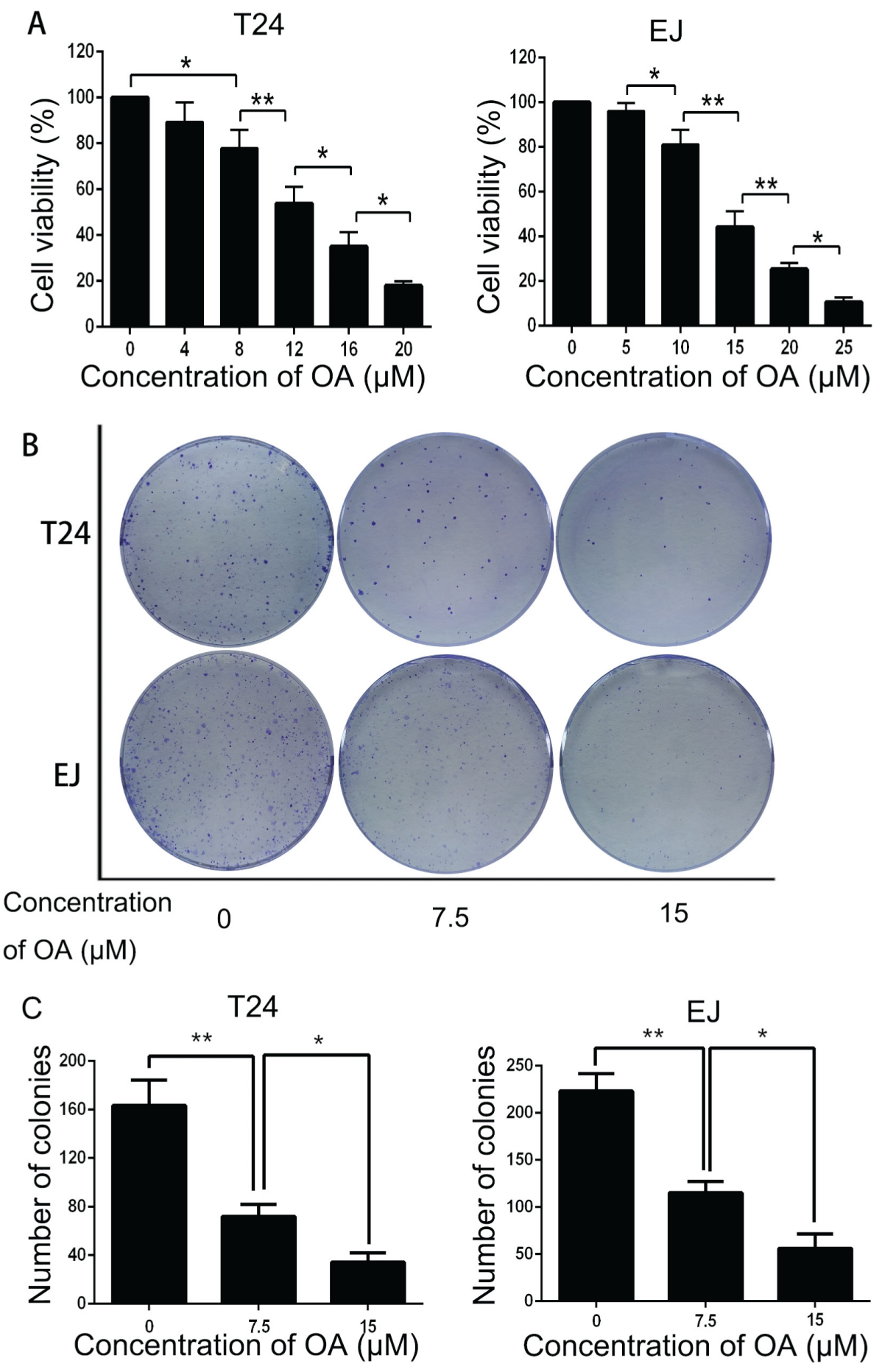

Figure 1: OA inhibited cell viability and proliferation in bladder cancer cells. (A) Cell viability of T24 and EJ cells. (B) and (C) Colony formation assay of T24 and EJ cells. The values were represented as mean $\pm \mathrm{SD}$ of three replicates. ${ }^{*} P<0.05$. ${ }^{* *} P<0.01$. 
Thr172 were determined. As shown in Figure 3C, OA treatment did not significantly alter the protein level of total AMPK $\alpha$, but increased P-AMPK $\alpha$ Thr172 in both T24 and EJ cells. These data indicated that OA might activate AMPK pathway in human bladder cancer cells. Previous study showed that AMPK could suppress mTOR and directly activate ULK1 by phosphorylating ULK1 at
Ser317, and finally enhanced autophagy [26]. We then examined several downstream targets of AMPK which are correlated to autophagy such as mTOR, p-mTOR Ser2448 and p-ULK1 Ser317 and the results exhibited that OA treatment did not change the protein level of total mTOR, but appreciably reduced expression of p-mTOR Ser2448 and increased p-ULK1 at Ser317. As a result, inhibition
A

T24

EJ

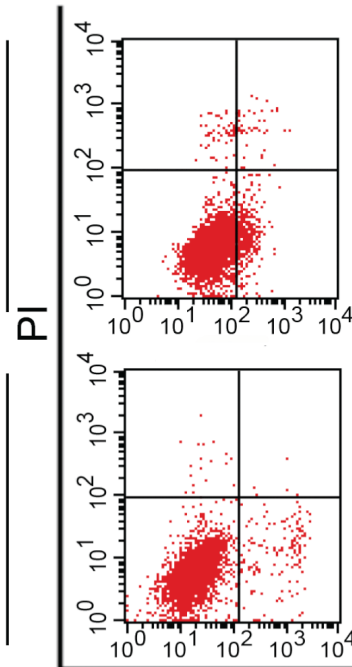

Concentration

of $O A(\mu M)$

B

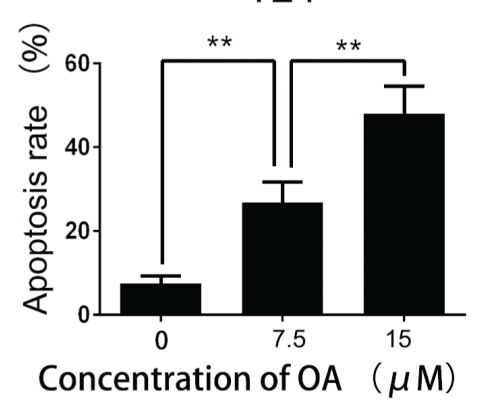

C

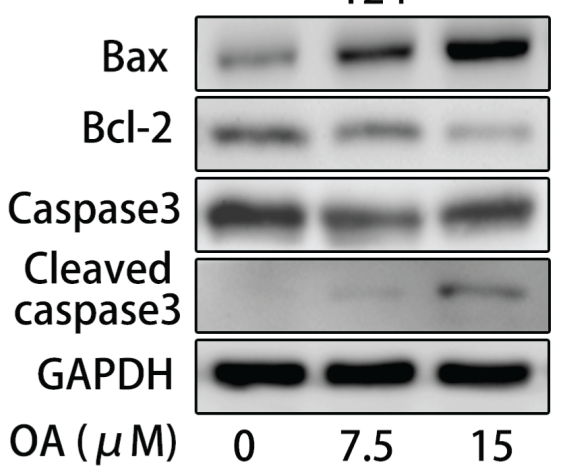

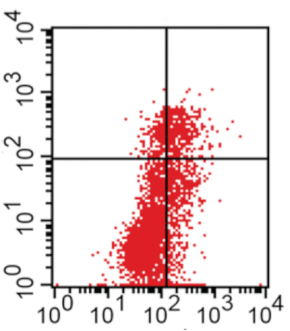
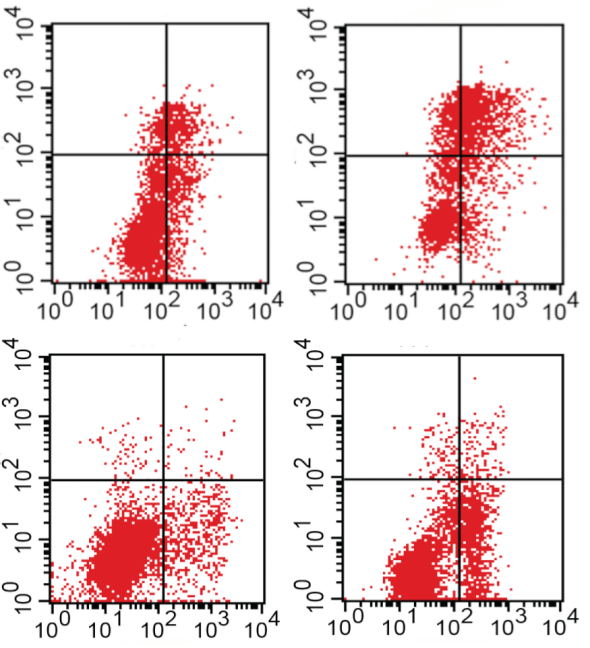

FITC

7.5

15

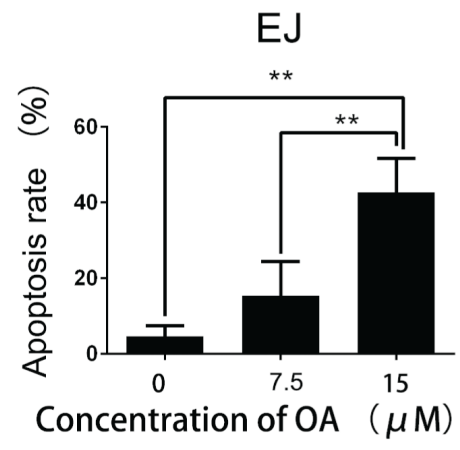

EJ

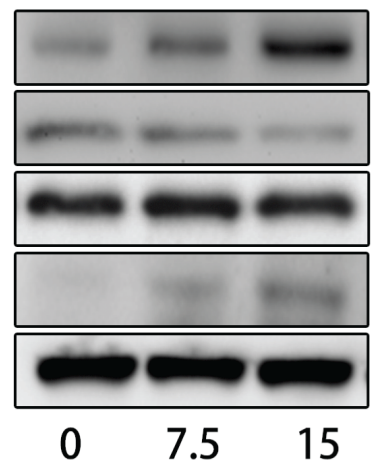

Figure 2: OA induced apoptosis in bladder cancer cells. (A) and (B) Cell apoptosis was measured by flow cytometry. The values were expressed as mean \pm SD of three independent experiments. (C) OA regulated the expression levels of apoptosis-related proteins in bladder cancer cells. Bax, bcl-2, caspase-3 and cleaved caspase-3 levels were detected by using Western blotting. GAPDH was used as a loading control. $* P<0.05$. $* * P<0.01$. 
of AMPK reduced the level of p-ULK1 at Ser317 and rescued p-mTOR Ser2448 upon OA treatment. These results indicated that $\mathrm{OA}$ might induce autophagy through activating AMPK-mTOR-ULK1 signaling pathway in human bladder cancer cells.

\section{Blocking autophagy enhanced OA-induced apoptosis in $\mathrm{T} 24$ cells}

To verify the effect of autophagy on OA-induced apoptosis, 3-MA (3-Methyladenine, a small molecule inhibitor of autophagy) was used to abolish autophagy in human bladder cancer cells. As shown in Figure 5A and $5 \mathrm{~B}, 3-\mathrm{MA}$ reduced the amount of both yellow puncta (autophagosomes) and red puncta (autophagolysosomes), indicating that 3-MA inhibited the formation of OA-induced autophagosomes. Cell viability was reduced synergistically when treated by OA plus 3-MA as compared treatment with one agent alone in T24 cells (Figure 5C). In line with this finding, Western blotting revealed that 3-MA diminished OA-induced LC3B-II expression. At the same time, 3-MA reduced the expression of anti-apoptotic protein Bcl-2 (Figure 5D). Furthermore, inhibition of autophagy with ATG7 siRNA and bafilomycin A1 also confirmed that blocking autophagy enhanced OA-induced apoptosis of bladder cancer cells (Figure 4). In general, our evidence supported the hypothesis that autophagy may compromise OA-induced cytotoxicity in human bladder cancer cells.
A

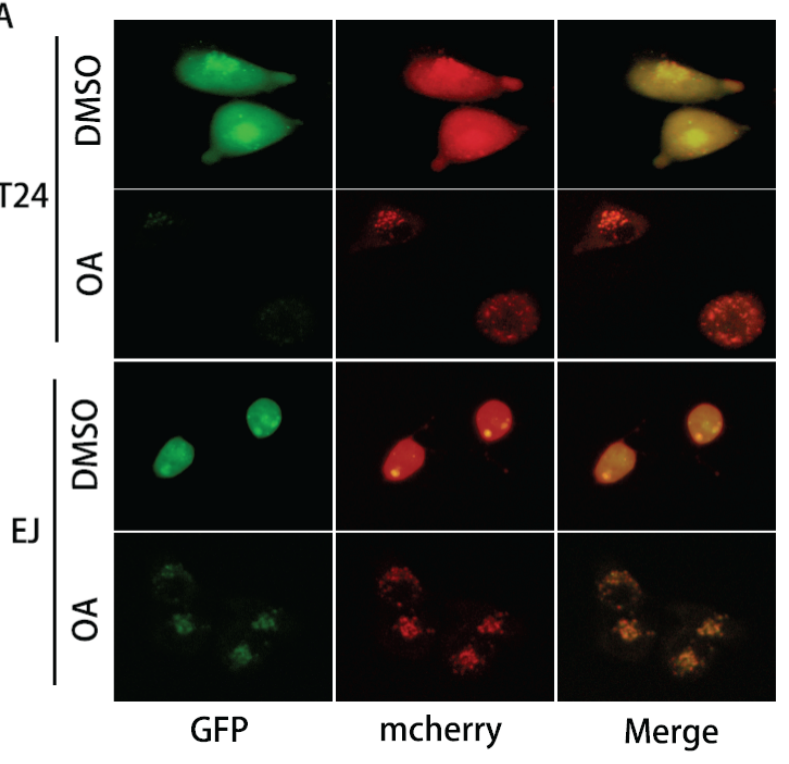

C

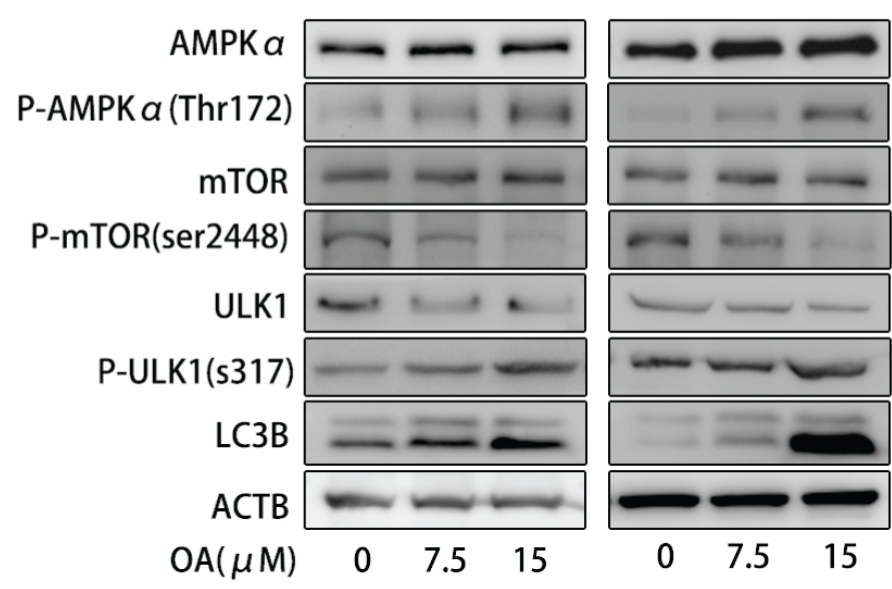

B

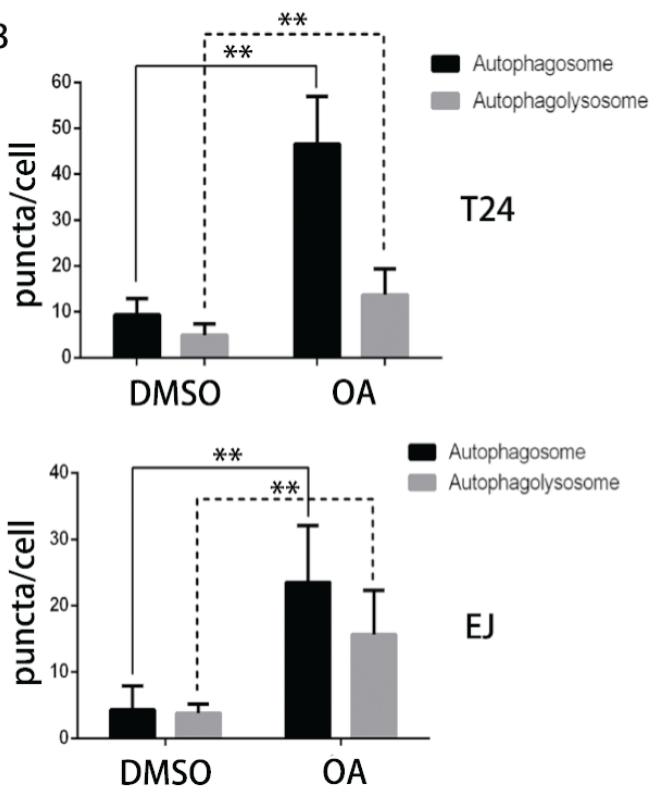

D

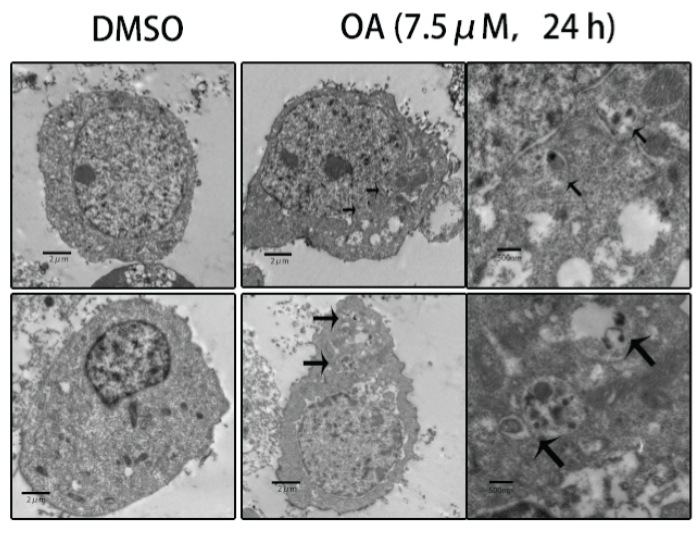

$\mathrm{T} 24$

EJ

Figure 3: OA induced autophagy through AMPK-mTOR-ULK1 axis in bladder cancer cells. (A) and (B) T24 and EJ pretransfected with adenovirus mCherry-GFP-LC3B were treated with DMSO (v/v, 1:1000) or OA (7.5 $\mu \mathrm{M})$ for $24 \mathrm{~h}$, then observed under a fluorescence microscope. (C) Expression of AMPK $\alpha$, P- AMPK $\alpha$ (Thr 172), mTOR, P-mTOR (Ser 2448), ULK-1, P-ULK1 (Ser 317), LC3B was detected by using Western blotting. (D) Autophagic vacuoles in T24 and EJ cells were examined under a transmission electron microscope (TEM). $* P<0.05 . * * P<0.01$. 


\section{Activating autophagy compromised OA-induced apoptosis of $\mathrm{T} 24$ cells}

To further validate our conclusion, we evaluated the effect of another autophagy promoter rapamycin (a specific mTOR inhibitor that induces autophagy) on OA-induced apoptosis. As expected, rapamycin increased the number of both yellow and red puncta, indicating that rapamycin could promote OA-induced autophagosomes formation (Figure 5A and 5B). Accordingly, rapamycin up-regulated OA-mediated LC3B-II formation, and increased cell viability dramatically in the absence of OA. Additionally, rapamycin, by enhancing autophagy, increased expression of Bcl-2 (Figure 5E and 5F). These findings further confirmed that activation of autophagy compromised OAinduced cytotoxicity in human bladder cancer cells.

\section{AMPK was implicated in protective autophagy upon OA-induced apoptosis in T24 cells}

To examine the effect of AMPK on OA-induced apoptosis, we employed dorsomorphin, an AMPK inhibitor to abolish OA-induced autophagy in bladder cancer cells. Our results showed that dorsomorphin substantially increased the number of both yellow and red puncta as compared with negative control group. Nonetheless, it decreased the number of both yellow and red puncta in those cells upon treatment with OA (Figure 6A and 6B). Dorsomorphin distinctly inhibited cell viability in the absence of OA (Figure 6C). As shown in Figure 6D, dorsomorphin down-regulated P-AMPK $\alpha$, P-ULK1 (s317), rescued the level of P-mTOR (s2448).

What is more, dorsomorphin up-regulated LC3B-II expression when compared with the negative control, while it attenuated OA-mediated increment of LC3B-II level. Even though dorsomorphin induced autophagy in T24 cells without OA treatment, it might enhance cytotoxicity by inhibiting AMPK. Then, we used AICAR to activate the AMPK to see if it exerts the similar effect, like an autophagy activator, and our results showed that AICAR increased the amount of yellow and red puncta and LC3B-II formation without OA treatment (Figure 6A and 6B), and AICAR reversed OA-induced inhibitory effect on cell viability in T24 cells (Figure 6E). Western blotting showed that AICAR promoted autophagy in the absence of OA by activating AMPK (Figure 6F).

\section{DISCUSSION}

Previous studies have demonstrated that OA triggered a wide array of biological processes in cancer cells, such as apoptosis and $\mathrm{G}_{0} / \mathrm{G}_{1}$ phase arrest [8]. This study demonstrated that OA could inhibit cell viability/ proliferation and induce apoptosis/autophagy in human bladder cancer cells. It is worthy mentioning that OAinduced autophagy per se compromised its cytotoxicity, and autophagy inhibitors, like 3-MA and bafilomycin A1, enhanced OA-mediated anti-cancer effect.

Inducing apoptosis has been used as an important strategy for cancer therapy. In current study, OA was found to be capable of inhibiting cell viability and proliferation, and, moreover, OA increased apoptosis in both T24 and EJ cells. Bax and Bcl-2 are crucial apoptotic regulators, and when $\mathrm{Bax}$ is in excess of $\mathrm{Bcl}-2$, the ensuring formation of homodimers (Bax-Bax) can promote apoptosis [27]. Cleaved caspase-3 is the mutual downstream effector of many apoptosis pathways, and acts as an executioner protease during apoptosis [28]. Our study showed that upon the administration of higher concentration OA, the protein level of Bax was increased, whereas Bcl-2 declined. After treatment with OA for $24 \mathrm{~h}$, OA cleaved apoptosis marker caspase- 3 in both cell lines. These data suggested that OA might induce apoptosis in bladder cancer cells through the mitochondrial pathway.

It has been reported that $\mathrm{OA}$ induced apoptosis and autophagy in a variety of cancer cells. OA could recruit LC3B-II formation [10-13], but the crosstalk between apoptosis and autophagy remains to be further clarified. Autophagy, an eukaryotic conserved catabolic process, has been deemed as a prerequisite for cancer development and potential target of cancer treatment due to its involvement with damnification, tumorigenesis and metabolic stress $[29,30]$. Multiple natural compounds, such as bergapten and ginsenoside Rh2, can induce autophagy in breast or hepatocellular carcinoma cells [31, 32]. It is known that LC3I converts to LC3II during the formation of double-membraned autophagosomes. Therefore, LC3II is generally considered as a marker of autophagy. Our present study showed that OA treatment conspicuously increased protein level of LC3B-II. Besides, in bladder cancer cells transfected with adenovirus containing LC3B, the number of autophagosomes and autophagolysosomes was increased significantly following $24 \mathrm{~h}$-treatment with OA. Transmission electron microscopy further verified the existence of autophagosomes in both T24 and EJ cells after treatement with $7.5 \mu \mathrm{M}$ of OA. All these findings suggested that OA might induce autophagy in human bladder cancer cells. Together with several other studies demonstrating that OA induced autophagy in hepatocellular carcinoma and gastric cancer cells $[11,12]$, OA might be able to induce autophagy in most tumor cells. AMPK is a cellular energy sensor conserved in all eukaryotic cells, and activation of AMPK can lead to dephosphorylation of mTOR and thereby enhance autophagy flux [33]. Our results exhibited that OA suppressed p-mTOR (Ser2448) and increased the levels of p-AMPK $\alpha$ (Thr172) and p-ULK1 (Ser317), indicating that AMPK-mTOR-ULK1 pathway might take part in OA-induced autophagy in bladder cancer cells.

Autophagy is a double-edged sword for the pharmacotherapy of malignancies. Some agents induce autophagic cell death, while others ellicit autophagy 


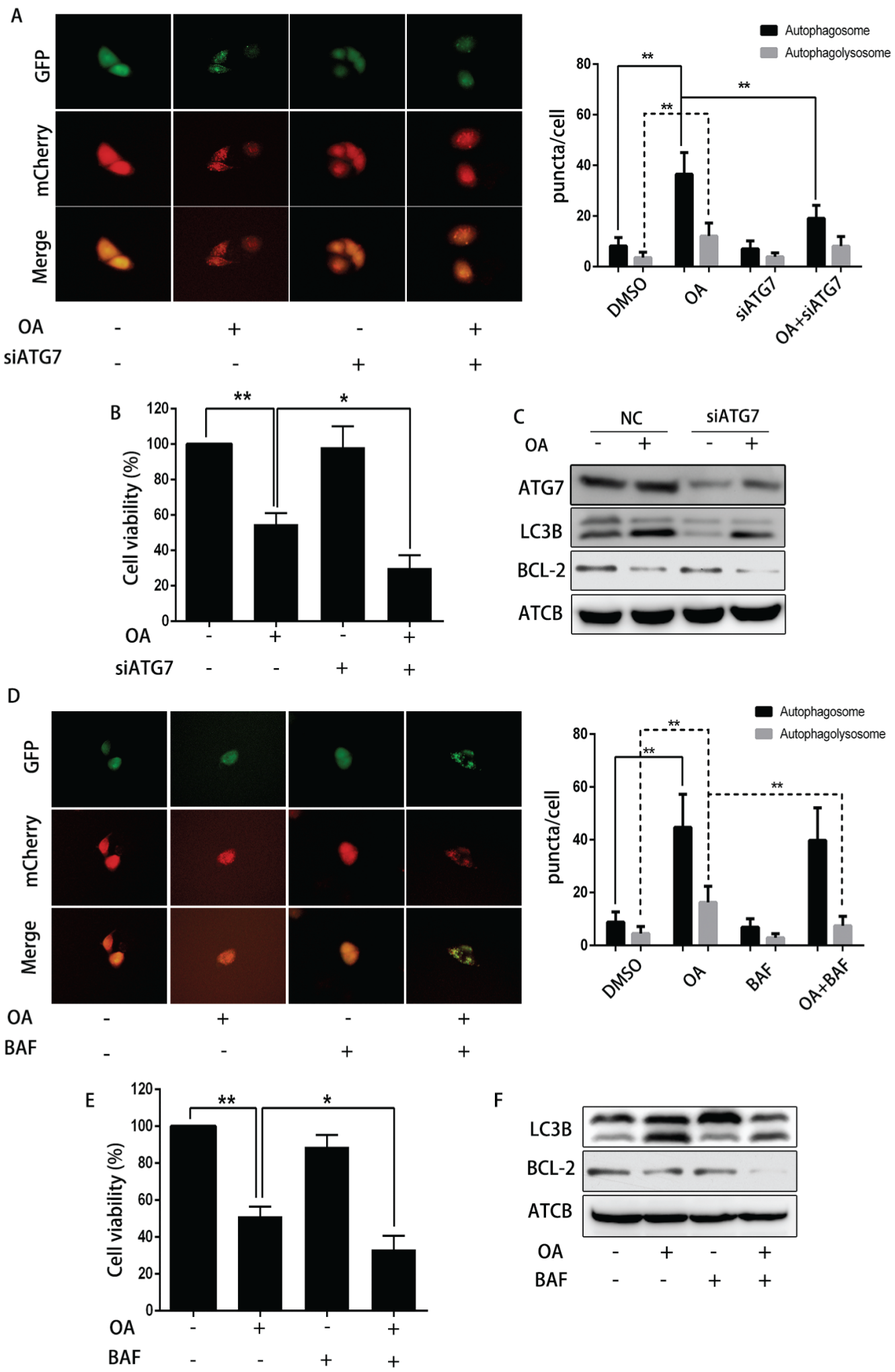

Figure 4: The impact of siRNA ATG7 or bafilomycin A1 on OA-induced anti-cancer effect. (A) T24 cells pre-transfected with adenovirus mCherry-GFP-LC3B were transfected with siRNA ATG7 or control siRNA for $72 \mathrm{~h}$, then incubated with DMSO (v/v, 1:1000) or OA $(7.5 \mu \mathrm{M})$ for another $24 \mathrm{~h}$. Cells were then imaged by fluorescence microscopy. (B) CCK8 assay was used to analyze the viability of T24 cells. (C) Western blotting was used to analyze the protein level of ATG7, LC3B and Bcl-2. (D) T24 cells pre-transfected with adenovirus mCherry-GFP-LC3B were pre-treated with bafilomycin $(100 \mathrm{nM})$ for $4 \mathrm{~h}$, then incubated with DMSO (v/v, 1:1000) or $\mathrm{OA}(7.5 \mu \mathrm{M})$ for another $24 \mathrm{~h}$. Cells were then imaged by fluorescence microscopy. (E) CCK8 assay was used to analyze the cell viability. (F) Western blotting was used to analyze the protein level of LC3B and Bcl-2. $* * P<0.05 . * * P<0.01$. 

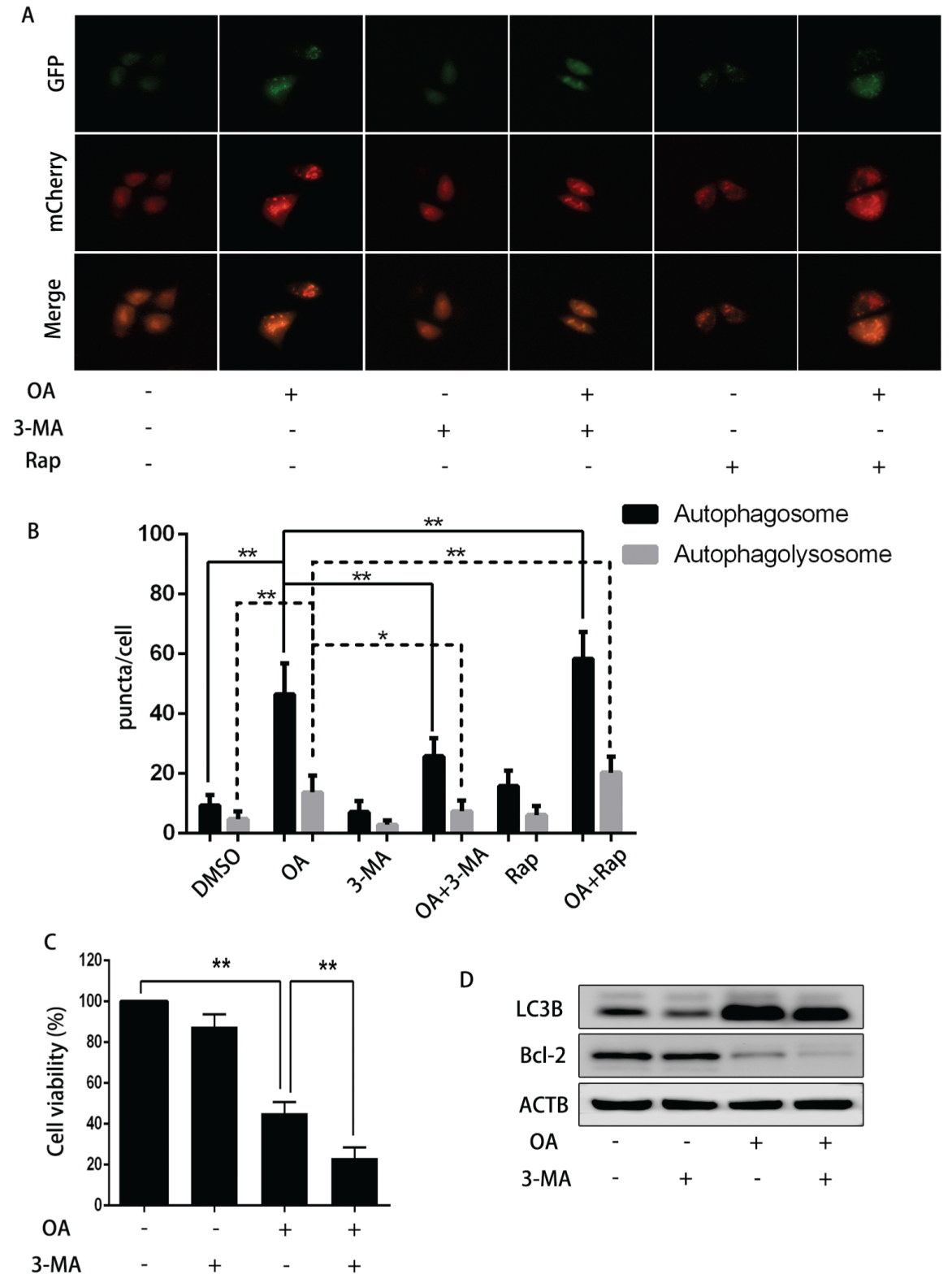

D

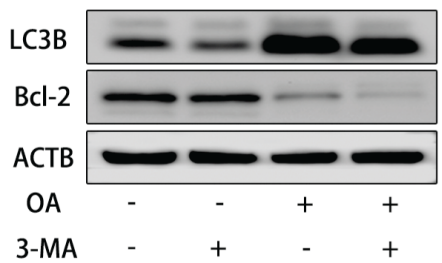

$\mathrm{E}$

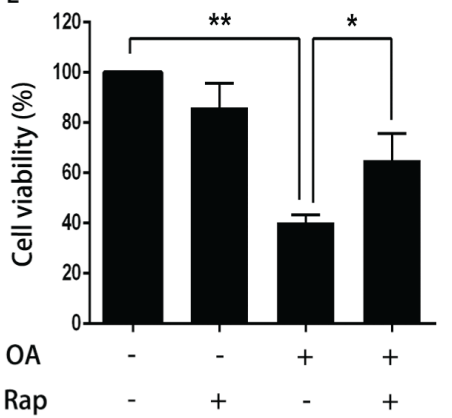

$\mathrm{F}$

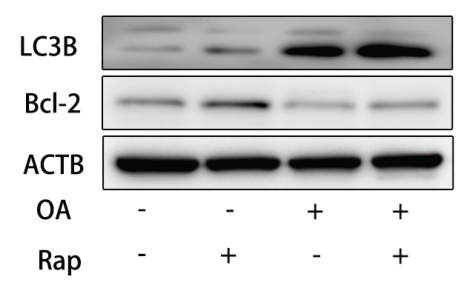

Figure 5: The impact of 3-MA or rapamycin on OA-induced anti-cancer effect. (A) and (B) T24 cells pre-transfected with adenovirus mCherry-GFP-LC3B were pre-treated with 3-MA $(5 \mathrm{mM})$ or rapamycin $(200 \mathrm{nM})$ for $2 \mathrm{~h}$, then incubated with DMSO (v/v, 1:1000) or OA $(7.5 \mu \mathrm{M})$ for another $24 \mathrm{~h}$. Cells were then imaged by fluorescence microscopy. (C) T24 cells pre-treated with or without 3-MA $(5 \mathrm{mM})$ for $2 \mathrm{~h}$ were treated with DMSO $(\mathrm{v} / \mathrm{v}, 1: 1000)$ or OA $(7.5 \mu \mathrm{M})$ for another $24 \mathrm{~h}$. CCK8 assay was used to analyze the cell viability. (D) Western blotting was used to analyze the protein level of LC3B and Bcl-2. (E) T24 cells pre-treated with or without rapamycin $(200 \mathrm{nM})$ for $2 \mathrm{~h}$ were treated with DMSO $(\mathrm{v} / \mathrm{v}, 1: 1000)$ or OA $(7.5 \mu \mathrm{M})$ for another $24 \mathrm{~h}$. CCK8 assay was used to analyze the cell viability. (F) Western blotting was employed to analyze the protein level of LC3B and Bcl-2. ${ }^{*} P<0.05 . * * P<0.01$. 
A

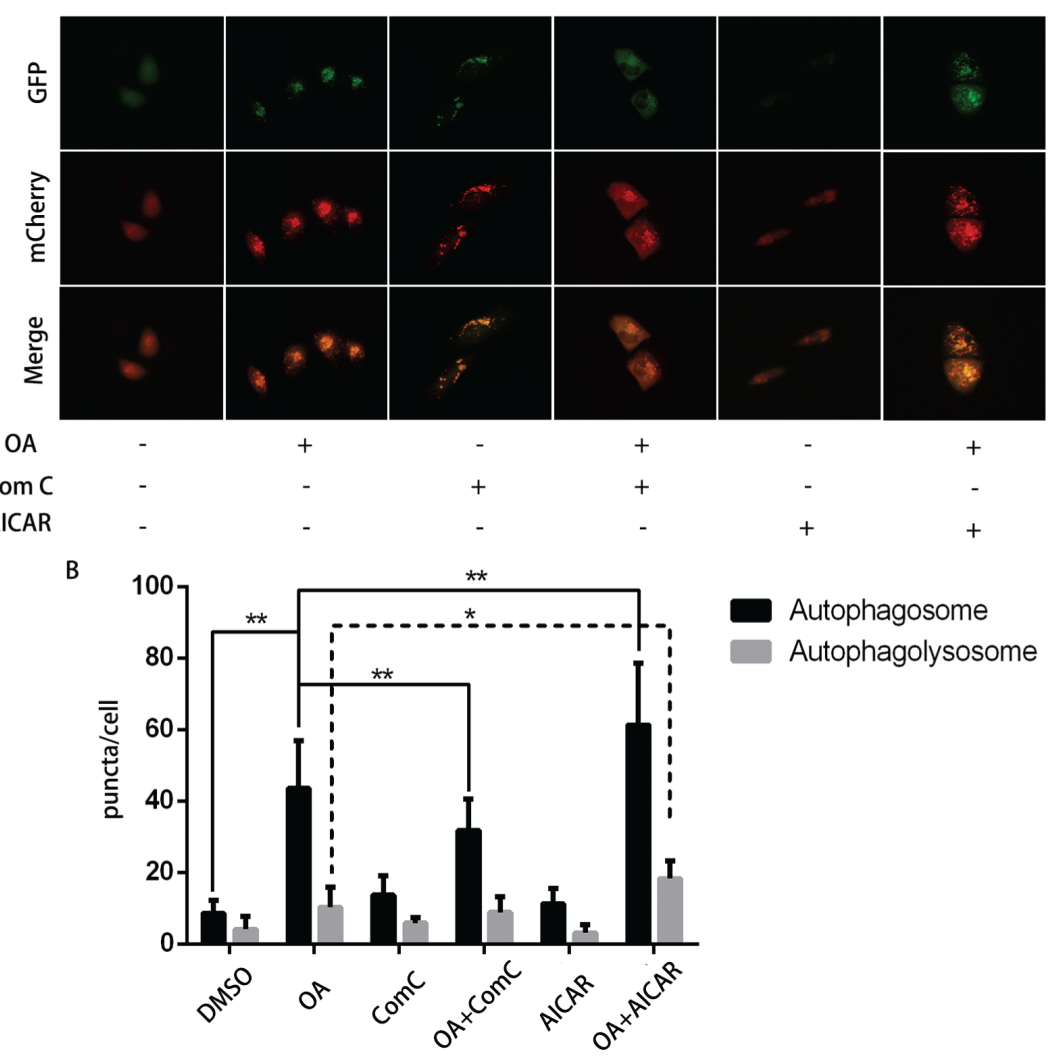

C

D
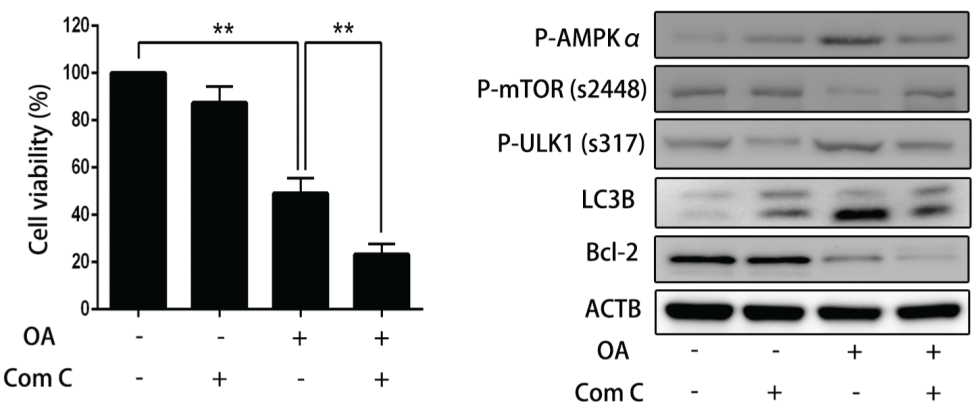

$E$

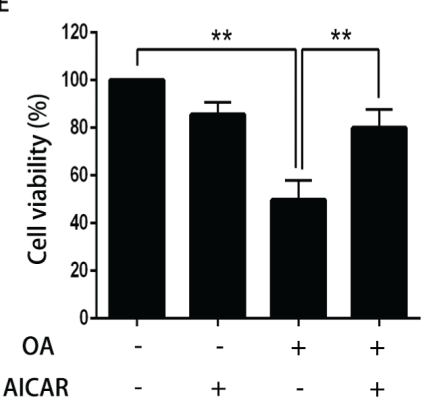

$\mathrm{F}$

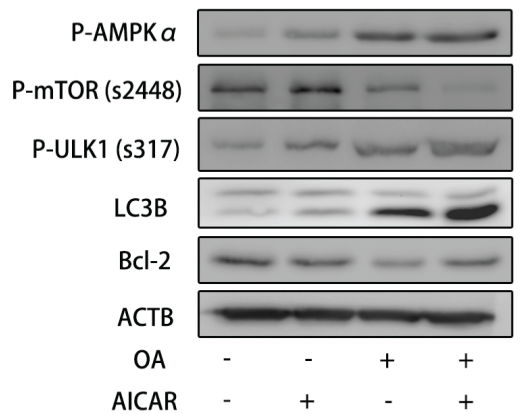

Figure 6: The impact of dorsomorphin or AICAR on OA-induced anti-cancer effect. (A) and (B) T24 cells pre-transfected with adenovirus mCherry-GFP-LC3B (10 MOI) for $24 \mathrm{~h}$ were pre-treated with dorsomorphin $(10 \mu \mathrm{M})$ or AICAR (400 $\mu \mathrm{M})$ for $2 \mathrm{~h}$, and then incubated with DMSO (v/v, 1:1000) or OA $(7.5 \mu \mathrm{M})$ for another $24 \mathrm{~h}$. Cells were then imaged by fluorescence microscopy. $* * P<0.05$. $* * P<0.01$. (C) T24 cells pre-treated with or without dorsomorphin $(10 \mu \mathrm{M})$ for $2 \mathrm{~h}$ were treated with DMSO $(\mathrm{v} / \mathrm{v}, 1: 1000)$ or OA $(7.5 \mu \mathrm{M})$ for another $24 \mathrm{~h}$. CCK8 assay were used to analyzed the cell viability. (D) Western blotting was used to analyze the protein level of LC3B and Bcl-2. (E) T24 cells pre-treated with or without AICAR $(400 \mu \mathrm{M})$ for $2 \mathrm{~h}$ were treated with DMSO (v/v, 1:1000) or OA $(7.5 \mu \mathrm{M})$ for another $24 \mathrm{~h}$. CCK8 assay was used to analyze the cell viability. (F) Western blotting was used to analyze the protein level of LC3B and Bcl-2. 
which work against their anti-cancer effects. For instance, bergapten [31], norcantharidin [34], hernandezine [35] have been reported to cause autophagic cell death, while berberine [36], 3,4,4-trihydroxy-trans-stilbene [37] induced autophagy as a protective mechanism against the anti-cancer agents. In this study, 3-MA, bafilomycin A1 and ATG7 siRNA were used to block OA- induced autophagy in T24 cells, and the results demonstrated that inhibition of autophagy significantly reduced cell viability. In contrast, rapamycin or AMPK activator AICAR markedly diminished OA-induced cell death.

To sum up, this study demonstrated that OA induced protective autophagy by activating AMPK-mTORULK1 pathway in bladder cancer cells, and combined use of autophagy inhibitors could enhance OA-induced anti-cancer effects. Our findings showed that OA in combination with autophagy inhibitors might be a novel alternative for the treatment of bladder cancer.

\section{MATERIALS AND METHODS}

\section{Cell culture}

T24 and EJ cell lines were cultured in Dulbecco's modified eagle medium (Gibco, Thermo Fisher Scientific Inc., Waltham, MA, USA) supplemented with 10\% fetal bovine serum (Gibco) in a $5 \% \mathrm{CO}_{2}$ humidified incubator at $37^{\circ} \mathrm{C}$.

\section{Reagents and antibodies}

Oleanolic acid (OA) was purchased from SigmaAldrich (Saint Louis, MO, USA), and was dissolved in dimethyl sulfoxide (Sigma-Aldrich, Saint Louis, MO, USA) at a stock concentration of $30 \mathrm{mM}$ and stored at $-20^{\circ} \mathrm{C}$. 3-methyladenine (S2767), rapamycin (S1039) dorsomorphin (compound C, S7306) and AICAR (Acadesine, S1802) were bought from Selleckchem (Huston, TX, USA). Bafolimycin A1 were procured from InvivoGen (San Diego, CA, USA). Cell Counting Kit-8 was from Dojindo (Kumamoto, Japan). FITC Annexin $\mathrm{V}$ Apoptosis Detection Kit I came from BD biosciences (\#556547, Franklin Lakes, NJ, USA). ATG7 siRNA I (6604S) and control siRNA (6568S) were products of Cell Signaling Technology (Beverly, MA, USA). Antibodies against mTOR (6308), phosphor-mTOR (Ser2448) (3308), caspase 3 (6311), cleaved-caspase 3 (7022) were all from Affinity Biosciences (Cincinnati, OH, USA). Antibodies against AMPK $\alpha$ (5831S), phospho-AMPK $\alpha$ (Thr172) (2535S), ULK1 (8084S), phospho-ULK1 (Ser317) (12753S), LC3B (3868S) were bought from Cell Signaling Technology. Antibodies against Bax (23931-1AP), BCL2 (12789-1-AP), GAPDH antibody (10494-1AP), $\beta$-actin antibody (20536-1-AP), AP conjugated goat anti-mouse and anti-rabbit secondary antibodies were from Proteintech (Chicago, IL, USA).

\section{Cell viability assay}

Cells (5000 cells per well) were plated into 96-well plate, cultured with completed medium, then treated with various dosages of OA or other reagents. $24 \mathrm{~h}$ after treatment, $10 \mu \mathrm{L} \mathrm{CCK}-8$ solution was added to each well and the plate was incubated for $2 \mathrm{~h}$ at $37^{\circ} \mathrm{C}$. The absorbance at $450 \mathrm{~nm}$ was measured on a microplate reader (Tecan, Mannedorf, Switzerland).

\section{Flow cytometry}

Cell apoptosis was detected using the FITC Annexin V Apoptosis Detection Kit I by following the manufacturer's protocols. Cells $\left(5 \times 10^{5}\right.$ per well $)$ were seeded into 6-well plate overnight and then treated with the indicated concentrations of OA for $24 \mathrm{~h}$. Cells were collected, suspended in Annexin V binding buffer, incubated with FITC Annexin V and propidium iodide (PI) and analyzed on a FACSVerse ${ }^{\mathrm{TM}}$ flow cytometer (BD Biosciences, San Jose, CA, USA).

\section{Colony formation assay}

Cells (1000 cells per well) were seeded into 6-well plate into 2-ml culture medium overnight. The medium was then changed with fresh medium containing OA (7.5 and $15 \mu \mathrm{M}$ ) or vehicle (DMSO) for 2 days. Subsequently, the medium was changed with fresh medium every 2 days for another 10 days. Colonies were fixed with $4 \%$ paraformaldehyde and stained with $0.1 \%$ crystal violet for $10 \mathrm{~min}$ respectively at room temperature. Colonies consisting of more than 50 cells were counted.

\section{Tandem mCherry-GFP fluorescence microscopy}

Cells $\left(1 \times 10^{4}\right.$ cells per well $)$ were seeded into 12 -well plate in $1 \mathrm{ml}$ culture medium overnight, then transfected with adenovirus mCherry-GFP-LC3B (10 MOI) for 24 hours. Cells were treated with or without OA $(7.5 \mu \mathrm{M})$ for another $24 \mathrm{~h}$. After treatment, the cells were washed with PBS three times and examined under an Olympus IX73 fluorescence microscope (Olympus, Japan). The numbers of GFP and mCherry dots were determined by fluorescent puncta then images were processed with cellSens Entry (Olympus). A total of more than 20 cells under each condition were counted for quantification of autophagy.

\section{RNA interference of ATG7}

T24 cells were seeded into 6 -well plates. The next day, when the cells reached a $40 \%$ confluency, $100 \mathrm{nM}$ ATG7 siRNA I or control siRNA and $5 \mu$ l Lipfectamine 2000 transfection reagent (Invitrogen, Carlsbad, CA, USA) were diluted with $250 \mu \mathrm{l}$ opti-MEM reduced-serum medium (Gibco, Grand Island, NY, USA) respectively. The medium was incubated for $5 \mathrm{~min}$ at room temperature. 
Afterwards, diluted si-RNAs were added into diluted Lipofectamine 2000 and incubated for $20 \mathrm{~min}$ at room temperature. $500 \mu \mathrm{l}$ of the mixture was added into each well. The medium was replaced with fresh medium after 6 hours.

\section{Western blotting}

Cells were lysed in RIPA buffer containing phenylmethanesulfonyl fluoride and phosphatase inhibitor. The concentration of the protein samples was analyzed by using BCA Protein Assay Kit (Beyotime, Shanghai, China). Equal amounts of proteins from each sample were separated by SDS-PAGE gels ranging from $8 \%$ to $15 \%$ and electrotransferred onto PVDF membranes (Merck Millipore, Billerica, MA, USA). After incubation with primary and secondary antibodies, the immunoblots were visualized by ECL solution on a BioSpectrum Imaging System (UVP, Upland, CA, USA). GAPDH and $\beta$-actin were used as the loading controls. The intensities of the bands were analyzed by using ImageJ.

\section{Transmission electron microscopy}

T24 and EJ cells were treated with or without $7.5 \mu \mathrm{M}$ of $\mathrm{OA}$ for $24 \mathrm{~h}$ following fixation with a solution containing $3 \%$ glutaraldehyde plus $2 \%$ paraformaldehyde in $0.1 \mathrm{~mol} / \mathrm{L}$ cacodylate buffer ( $\mathrm{pH} 7.4$ ) at room temperature for $2 \mathrm{~h}$. The samples were then post-fixed, dehydrated, embedded, sectioned and double-stained with uranyl acetate and lead citrate, and electron micrographs were taken by employing a Trans-mission Electron Microscope (TECNAI G2 20 TWIN, FEI, Hillsboro, USA).

\section{Statistical analysis}

All experiments were performed at least three times. Data were presented as the mean \pm standard deviation (SD). Differences between experimental groups were analyzed by ultilizing one-way or two-way ANOVA. A $P$ value $<0.05$ was considered to be statistically significant.

\section{Abbreviations}

OA, oleanolic acid; AMPK, AMP-activated protein kinase; $\mathrm{mTOR}$, mechanistic target of rapamycin; ULK1, unc-51 like autophagy activating kinase 1; ATG7, autophagy related 7; 3-MA, 3-methyladenine; AICAR, acadesine; muscle-invasive bladder cancer, MIBC; BECN1, beclin1; PI3K, phosphatidylinositol 3-kinase; AKT, serine/threonine kinase 1; GSK3 $\beta$, glycogen synthase kinase 3 beta; ERK1/2, mitogen-activated protein kinase; eEF2K, eukaryotic elongation factor 2 kinase; BAX, BCL2 associated X, apoptosis regulator; BCL-2, B cell leukemia/lymphoma 2; TEM, transmission electron microscopy; LC3B, microtubule associated protein 1 light chain 3 beta

\section{ACKNOWLEDGMENTS}

We are indebted to Dr. Fuqing Zeng (Department of Urology, Union Hospital, Tongji Medical College, Huazhong University of Science and Technology) for providing some of the cell lines used in this project.

\section{CONFLICTS OF INTEREST}

The authors declare no conflicts of interest.

\section{FUNDING}

This study was funded by the National Natural Science Foundation of China (Nos. 81272847 and 30973008) and the Program for New Century Excellent Talents in University (NCET-13-0239) awarded to Yifei Xing.

\section{REFERENCES}

1. Miller KD, Siegel RL, Lin CC, Mariotto AB, Kramer JL, Rowland JH, Stein KD, Alteri R, Jemal A. Cancer treatment and survivorship statistics, 2016. CA Cancer J Clin. 2016; 66:271-89. doi: 10.3322/caac.21349.

2. Stein JP, Skinner DG. Radical cystectomy for invasive bladder cancer: long-term results of a standard procedure. World J Urol. 2006; 24:296-304. doi: 10.1007/s00345-0060061-7.

3. Pollier J, Goossens A. Oleanolic acid. Phytochemistry. 2012; 77:10-5. doi: 10.1016/j.phytochem.2011.12.022.

4. Castellano JM, Guinda A, Delgado T, Rada M, Cayuela JA. Biochemical basis of the antidiabetic activity of oleanolic acid and related pentacyclic triterpenes. Diabetes. 2013; 62:1791-9. doi: 10.2337/db12-1215.

5. Amara S, Zheng M, Tiriveedhi V. Oleanolic Acid Inhibits High Salt-Induced Exaggeration of Warburg-like Metabolism in Breast Cancer Cells. Cell Biochem Biophys. 2016; 74:427-34. doi: 10.1007/s12013-016-0736-7.

6. Li HF, Wang XA, Xiang SS, Hu YP, Jiang L, Shu YJ, Li ML, Wu XS, Zhang F, Ye YY, Weng H, Bao RF, Cao Y, et al. Oleanolic acid induces mitochondrial-dependent apoptosis and G0/G1 phase arrest in gallbladder cancer cells. Drug Des Devel Ther. 2015; 9:3017-30. doi: 10.2147/ DDDT.S84448.

7. Luo Y, Liu Z, Zhang X, Huang J, Yu X, Li J, Xiong D, Sun $\mathrm{X}$, Zhong Z. Effect of a controlled-release drug delivery system made of oleanolic acid formulated into multivesicular liposomes on hepatocellular carcinoma in vitro and in vivo. Int J Nanomedicine. 2016; 11:3111-29. doi: 10.2147/IJN.S108445. 
8. Li X, Song Y, Zhang P, Zhu H, Chen L, Xiao Y, Xing Y. Oleanolic acid inhibits cell survival and proliferation of prostate cancer cells in vitro and in vivo through the PI3K/ Akt pathway. Tumour Biol. 2016; 37:7599-613. doi: 10.1007/s13277-015-4655-9.

9. Mu DW, Guo HQ, Zhou GB, Li JY, Su B. Oleanolic acid suppresses the proliferation of human bladder cancer by Akt/mTOR/S6K and ERK1/2 signaling. Int J Clin Exp Pathol. 2015; 8:13864-70.

10. Liu J, Zheng L, Ma L, Wang B, Zhao Y, Wu N, Liu G, Lin X. Oleanolic acid inhibits proliferation and invasiveness of Kras-transformed cells via autophagy. J Nutr Biochem. 2014; 25:1154-60. doi: 10.1016/j.jnutbio.2014.06.006.

11. Shi Y, Song Q, Hu D, Zhuang X, Yu S, Teng D. Oleanolic acid induced autophagic cell death in hepatocellular carcinoma cells via PI3K/Akt/mTOR and ROS-dependent pathway. Korean J Physiol Pharmacol. 2016; 20:237-43. doi: 10.4196/kjpp.2016.20.3.237.

12. Nie H, Wang Y, Qin Y, Gong XG. Oleanolic acid induces autophagic death in human gastric cancer cells in vitro and in vivo. Cell Biol Int. 2016; 40:770-8. doi: 10.1002/ cbin. 10612 .

13. Liu J, Zheng L, Zhong J, Wu N, Liu G, Lin X. Oleanolic acid induces protective autophagy in cancer cells through the JNK and mTOR pathways. Oncol Rep. 2014; 32:567-72. doi: 10.3892/or.2014.3239.

14. He C, Klionsky DJ. Regulation mechanisms and signaling pathways of autophagy. Annu Rev Genet. 2009; 43:67-93. doi: 10.1146/annurev-genet-102808-114910.

15. Kubisch J, Turei D, Foldvari-Nagy L, Dunai ZA, Zsakai L, Varga M, Vellai T, Csermely P, Korcsmaros T. Complex regulation of autophagy in cancer - integrated approaches to discover the networks that hold a double-edged sword. Semin Cancer Biol. 2013; 23:252-61. doi: 10.1016/j. semcancer.2013.06.009.

16. Liang XH, Jackson S, Seaman M, Brown K, Kempkes B, Hibshoosh H, Levine B. Induction of autophagy and inhibition of tumorigenesis by beclin 1. Nature. 1999; 402:672-6. doi: 10.1038/45257.

17. Aita VM, Liang XH, Murty VV, Pincus DL, Yu W, Cayanis E, Kalachikov S, Gilliam TC, Levine B. Cloning and genomic organization of beclin 1, a candidate tumor suppressor gene on chromosome 17q21. Genomics. 1999; 59:59-65. doi: 10.1006/geno.1999.5851.

18. Mathew R, Kongara S, Beaudoin B, Karp CM, Bray K, Degenhardt K, Chen G, Jin S, White E. Autophagy suppresses tumor progression by limiting chromosomal instability. Genes Dev. 2007; 21:1367-81. doi: 10.1101/ gad.1545107.

19. Mathew R, Karp CM, Beaudoin B, Vuong N, Chen G, Chen HY, Bray K, Reddy A, Bhanot G, Gelinas C, Dipaola RS, Karantza-Wadsworth V, White E. Autophagy suppresses tumorigenesis through elimination of p62. Cell. 2009; 137:1062-75. doi: 10.1016/j.cell.2009.03.048.
20. Rabinowitz JD, White E. Autophagy and metabolism. Science. 2010; 330:1344-8. doi: 10.1126/science.1193497.

21. Liu B, Cheng Y, Liu Q, Bao JK, Yang JM. Autophagic pathways as new targets for cancer drug development. Acta Pharmacol Sin. 2010; 31:1154-64. doi: 10.1038/aps.2010.118.

22. Cheng Y, Ren X, Hait WN, Yang JM. Therapeutic targeting of autophagy in disease: biology and pharmacology. Pharmacol Rev. 2013; 65:1162-97. doi: 10.1124/ pr.112.007120.

23. Sanduja S, Feng Y, Mathis RA, Sokol ES, Reinhardt F, Halaban R, Gupta PB. AMPK promotes tolerance to Ras pathway inhibition by activating autophagy. Oncogene. 2016; 35:5295-5303. doi: 10.1038/onc.2016.70.

24. Ji MM, Wang L, Zhan Q, Xue W, Zhao Y, Zhao X, Xu PP, Shen Y, Liu H, Janin A, Cheng S, Zhao WL. Induction of autophagy by valproic acid enhanced lymphoma cell chemosensitivity through HDAC-independent and IP3mediated PRKAA activation. Autophagy. 2015; 11:2160-71. doi: 10.1080/15548627.2015.1082024.

25. Gallo C, Dallaglio K, Bassani B, Rossi T, Rossello A, Noonan DM, D'Uva G, Bruno A, Albini A. Hop derived flavonoid xanthohumol inhibits endothelial cell functions via AMPK activation. Oncotarget. 2016; 7:59917-59931. doi: 10.18632/oncotarget.10990.

26. Feng Y, Yao Z, Klionsky DJ. How to control self-digestion: transcriptional, post-transcriptional, and post-translational regulation of autophagy. Trends Cell Biol. 2015; 25:354-63. doi: 10.1016/j.tcb.2015.02.002.

27. Zhou S, Wang Y, Zhu JJ. Simultaneous Detection of Tumor Cell Apoptosis Regulators Bcl-2 and Bax through a DualSignal-Marked Electrochemical Immunosensor. ACS Appl Mater Interfaces. 2016; 8:7674-82. doi: 10.1021/ acsami.6b01010.

28. Chen J, Mehta JL, Haider N, Zhang X, Narula J, Li D. Role of caspases in Ox-LDL-induced apoptotic cascade in human coronary artery endothelial cells. Circ Res. 2004; 94:370-6. doi: 10.1161/01.RES.0000113782.07824.BE.

29. He JD, Wang Z, Li SP, Xu YJ, Yu Y, Ding YJ, Yu WL, Zhang RX, Zhang HM, Du HY. Vitexin suppresses autophagy to induce apoptosis in hepatocellular carcinoma via activation of the JNK signaling pathway. Oncotarget. 2016; 7:84520-84532. doi: 10.18632/oncotarget.11731.

30. Silva LM, Jung JU. Modulation of the autophagy pathway by human tumor viruses. Semin Cancer Biol. 2013; 23:323-8. doi: 10.1016/j.semcancer.2013.05.005.

31. De Amicis F, Aquila S, Morelli C, Guido C, Santoro M, Perrotta I, Mauro L, Giordano F, Nigro A, Ando S, Panno ML. Bergapten drives autophagy through the upregulation of PTEN expression in breast cancer cells. Mol Cancer. 2015; 14: 130. doi: 10.1186/s12943-015-0403-4.

32. Yang Z, Zhao T, Liu H, Zhang L. Ginsenoside Rh2 inhibits hepatocellular carcinoma through beta-catenin and autophagy. Sci Rep. 2016; 6:19383. doi: 10.1038/srep19383. 
33. Sanduja S, Feng Y, Mathis RA, Sokol ES, Reinhardt F, Halaban R, Gupta PB. AMPK promotes tolerance to Ras pathway inhibition by activating autophagy. Oncogene. 2016; 35:5295-303. doi: 10.1038/onc.2016.70.

34. Xiao W, Dai B, Zhu Y, Ye D. Norcantharidin induces autophagy-related prostate cancer cell death through Beclin-1 upregulation by miR-129-5p suppression. Tumour Biol. 2015 Dec 5. doi: 10.1007/s13277-015-4488-6. [Epub ahead of print].

35. Law BY, Mok SW, Chan WK, Xu SW, Wu AG, Yao XJ, Wang JR, Liu L, Wong VK. Hernandezine, a novel AMPK activator induces autophagic cell death in drug-resistant cancers. Oncotarget. 2016; 7:8090-104. doi: 10.18632/ oncotarget. 6980 .
36. Wang J, Qi Q, Feng Z, Zhang X, Huang B, Chen A, Prestegarden L, Li X, Wang J. Berberine induces autophagy in glioblastoma by targeting the AMPK/mTOR/ULK1pathway. Oncotarget. 2016; 7:66944-66958. doi: 10.18632/ oncotarget.11396.

37. Zhang L, Dai F, Sheng PL, Chen ZQ, Xu QP, Guo YQ. Resveratrol analogue 3,4,4'-trihydroxy-trans-stilbene induces apoptosis and autophagy in human non-smallcell lung cancer cells in vitro. Acta Pharmacol Sin. 2015; 36:1256-65. doi: 10.1038/aps.2015.46. 